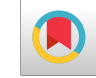

\title{
Investigating Therapeutic Effects of Retinoic Acid on Thyroid Cancer via Protein-Protein Interaction Network Analysis
}

\author{
Majid Rezaei-Tavirani (iD ${ }^{1}$, Mostafa Rezaei-Tavirani (iD) ${ }^{2}$ and Mona Zamanian Azodi (iD) ${ }^{2,}$ \\ ${ }^{1}$ Faculty of Medicine, Iran University of Medical Sciences, Tehran, Iran \\ ${ }^{2}$ Proteomics Research Center, Shahid Beheshti University of Medical Sciences, Tehran, Iran \\ "Corresponding author: Proteomics Research Center, Shahid Beheshti University of Medical Sciences, Tehran, Iran. Email: mona.azodi@gmail.com \\ Received 2019 April 20; Revised 2019 August 11; Accepted 2019 August 11.
}

\begin{abstract}
Background: Thyroid cancer is the most malignant type of endocrine tumor. The molecular investigation of applied treatments for this type of neoplasm could provide a better understanding of their mechanisms of action.

Objectives: Here, the differentially expressed proteins from a 2D gel-based proteomics of thyroid cancer cells treated by retinoic acid (RA) were considered for protein-protein interaction (PPI) network analysis.

Methods: Eight proteins related to the thyroid cancer cell line (FTC-133) treated with RA were extracted from an investigation by Trojanowicz et al. The query proteins and 50 neighbors were interacted by Cytoscape software via the STRING database. The network was analyzed, and hub-bottlenecks were identified. GeneMANIA determined the relationship between hub-bottlenecks. Protein complex analysis was done via MCODE. ClueGO + CluePedia was used to analyze gene ontology enrichment for hub-bottlenecks, differentially expressed hub-bottlenecks and the central cluster of the constructed network.

Results: GAPDH, ENO1, and PKM proteins as hub-bottleneck and PDHB as the seed protein of the main protein complex were introduced as central proteins. However, the query proteins were not included oncogenic proteins, several oncogene genes such as MYC, STAT3, and AKT1among neighbor proteins were connected to the query proteins. The Glycolytic process through fructose-6phosphate was the leading group of biological processes that were related to the central proteins.

Conclusions: It can be concluded that retinoic acid suppressed the activated glycolysis in thyroid cancer cells. The finding can be useful in the follow-up of patients. Additionally, RA regulates many oncogenes that act as a regulator of the determined central proteins.
\end{abstract}

Keywords: Proteomic Analysis, Protein-Protein Interaction Network, Retinoic Acid Therapy, Thyroid Cancer

\section{Background}

Of endocrine malignancies, thyroid cancer is the most common type that consists of papillary, follicular, medullary, and anaplastic forms originating from histopathological characteristics $(1,2)$. Most of its types are not manageable due to their resistance to nature towards conventional treatment methods (3). Moreover, it possesses the highest rate of mortality among endocrine cancers (4). The application of different approaches for thyroid cancer is available as thyroidectomy, radioactive iodine therapy, and thyroid-stimulating hormone suppression (5). On the other hand, retinoic acid (RA) has been identified as a potential anticancer agent for malignancies treatment such as neoplasms of thyroid, breast, lung, liver, and so on $(5,6)$. This vitamin A (retinol) metabolite plays meaningful participation in cell developmental and differential processes (6). Molecular research could guide substantially clinical approaches by introducing diagnostic and therapeutic biomarkers and drug targets that play critical roles in disease management. Proteomics, as one of the promising ones, can elaborate on a particular condition such as disease by the recognition of specific proteins related to that condition. These proteins, as the functional parts of the cells, can be examined for different aspects, including their expression profile and interactions with other proteins and molecules. In the cancer field, it is also feasible to identify biomarker candidates through proteome profiling for diagnosis and drug targeting approaches (7). There are many related proteomics studies in the investigation of the therapeutic effects of different agents on tumors $(8,9)$. These agents could interfere with the expression levels of many targets that can be quantified by techniques such as $2 \mathrm{D}$ gel electrophoresis-based proteomics. Thyroid cancer is not an exception in a study by Trojanowicz et al. (5) some proteins were introduced as 
the targets of RA effects on thyroid cancerous. In this respect, 8 proteins were proposed as the part of mechanisms, by which RA influenced on thyroid cancer (5). On the other hand, bioinformatics as a relatively new discipline models different types of diseases. Interaction network analysis is one of these ways that identifies the central proteins of an interesting map (10). These elements with the highest scores of interactions could play a significant role in the network strength and, consequently, valuable targets for designing appropriate drugs (11). Therefore, in a proteomics study, it is possible to filter and priorities the differentially expressed proteins based on interactions. These nodes can be more essential as biomarkers of specific conditions such as applied treatments in a disease status (12).

\section{Objectives}

Hence, to get a better insight into how retinoic acid impacts the cancer of the thyroid, a protein-protein interaction (PPI) network analysis is conducted in this research. In this way, some proteins could be highlighted as the most crucial agents that could be applied in clinical studies.

\section{Methods}

Eight proteins were extracted from a proteomics study entitled "Proteomic approach reveals novel targets for retinoic acid-mediated therapy of thyroid carcinoma" by Trojanowicz et al. These proteins include Enolase 1 (ENO1), glyceraldehyde-3-phosphate dehydrogenase (GAPDH), pyruvate kinase isoenzymes (PKM1/M2), transketolase (TKT), glutathione S-transferase P (GSTP1), peroxiredoxin 2 (PRDX2), annexin A2 (ANXA2), and peptidylprolyl cis-trans isomerase A (PPIA). In the mentioned research, the introduced proteins were identified as downregulated in the thyroid cancer cell line (FTC-133) treated with retinoic acid (5). In the present study, a PPI network analysis of these proteins is handled to understand the most novel proteins in terms of interaction patterns. Cytoscape software version 3.7.1 and its integrated plug-ins were used to construct and analyze the PPI map. At first, the mentioned proteins were queried by STRING DB v.1.4.2 (https://string-db.org/) $(13,14)$. Two networks, including one with just the searched proteins and the other one with the neighbor proteins, were retrieved. The statistical aspects are confidence score cut-off of 0.4 as the default option and addition of 50 surrounding proteins (for the second network). STRING DB can provide data from 4 sources, namely STRING PubMed, STRING Protein, STRING Diseases, and STITCH. STRING Protein source was used for obtaining the two constructed networks. The network, including additional 50 neighbors, was analyzed for the centrality parameters (degree $[\mathrm{K}]$ and betweenness centrality [BC]) by Network Analyzer software (15). GeneMANIA (http://www.genemania.org/plugin/), then, analyzed the prediction of the biological function between differentially expressed hub-bottlenecks (nodes with the highest value of degree and betweenness). This Cytoscape application provides interaction data types from different databases (16). Gene ontology analysis is a way to understand the biological process (BP), cellular component (CC), and molecular function (MF) of the interest set of genes or proteins. Biological processes for hub-bottlenecks differentially expressed hub-bottlenecks, and the central cluster of the constructed network was assessed via ClueGO + CluePedia. The statistical parameters assigned for these analyses were as grouping score cut-off of 0.5 , GO term min number of genes $=3$, and min gene percentage in term $=4$. The correction method for the P-value also was Bonferroni step down, and the two-sided hypergeometric test is the enrichment/depletion $(17,18)$.

\section{Results}

The 8 candidate genes were queried against the $\mathrm{Cy}$ toscape and STRING DB together, and the result showed 19 links among nodes (see the left side of Figure 1). Adding 50 relevant genes results in a network with 58 nodes and 981 edges (right side of Figure 1).

For assessing the hub-bottlenecks, the top $20 \%$ of nodes based on degree value as hubs and $20 \%$ of nodes based on betweenness as bottlenecks were considered. Common hubs and bottlenecks were identified as hubbottlenecks that are tabulated in Table 1. Eight proteins in the constructed network of RA affecting thyroid cancer cells could be understood as hub-bottlenecks. Among these proteins, some of the investigated DEPs, including GAPDH, ENO1, and PKM, are top hub-bottlenecks.

GeneMANIA analysis revealed relations between DE Hub-bottlenecks that are physical interactions, coexpression, predicted function, and co-localization that are assigned in different colors (Figure 2). The highest score is for physical interactions (ENO1 and GAPDH) (GAPDH and PKM), which is 67.64. The next ranked interaction is co-expression (ENO1, GAPDH, and PKM) with a score of 13.50. The later one is between ENO1 and GAPDH, which are co-localized. Predicted action is as well valid for all 3 proteins.

As it is illustrated in Figure 3, 3 complexes are obtained from the network. Four DEPs, including PKM, GAPDH, ENO1, and TKT are presented in the first complex. This cluster has 31 nodes and 457 edges. The seed protein in this protein complex is PDHB. The next cluster contains 19 and 139 

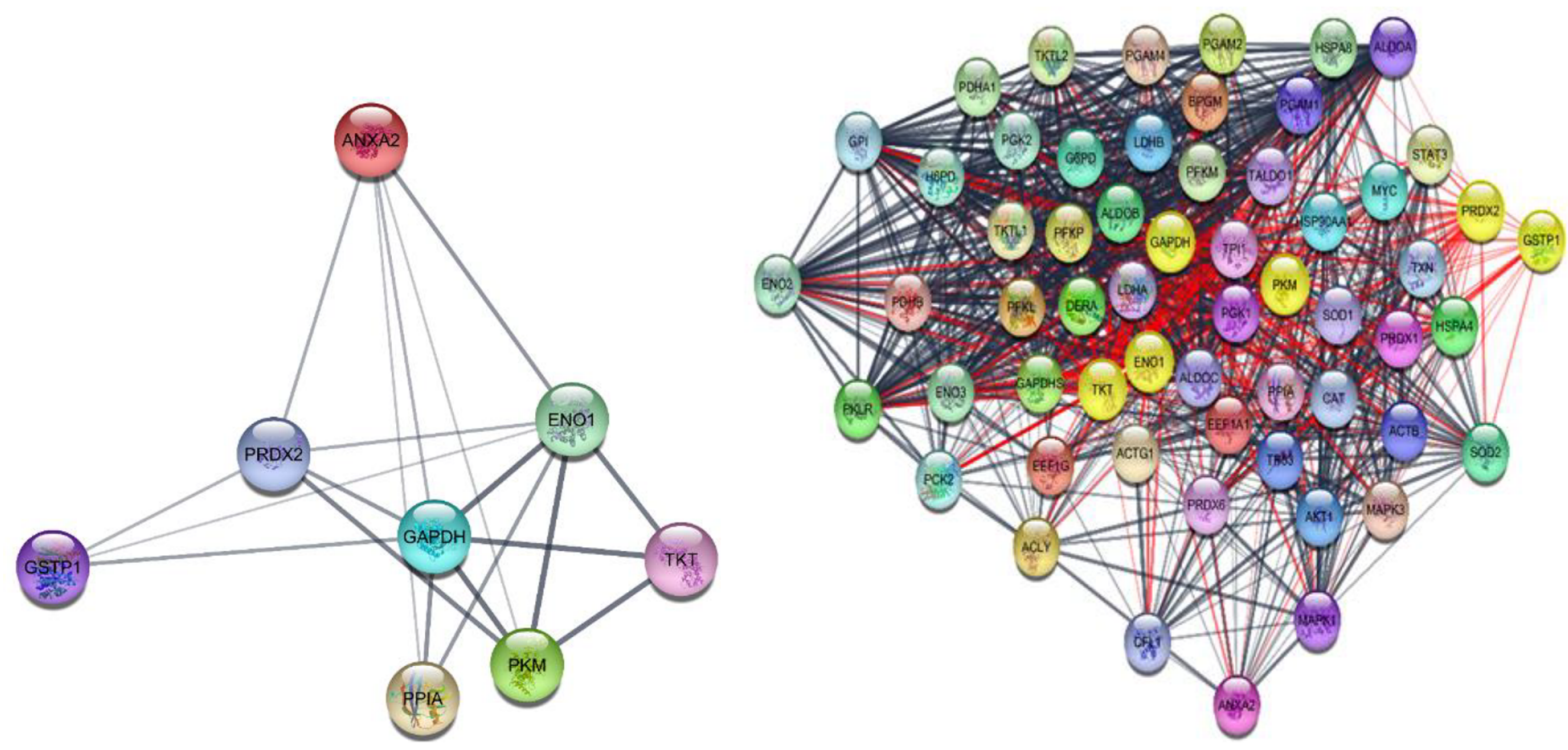

Figure 1. The interaction pattern of queried proteins indicates 8 nodes and 19 interactions among them (left side) and the network, including 8 query proteins and 50 additional relevant proteins (right side). The query proteins in the right network are shown in yellow color. The 981 edges connect 58 nodes.

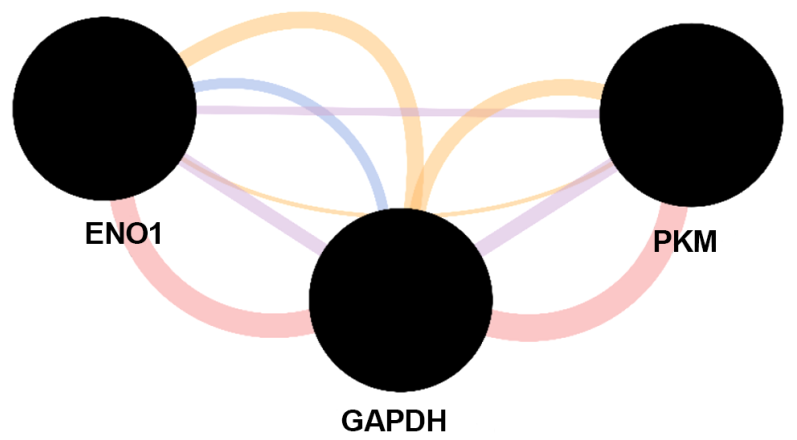

$\checkmark$ Physical Interactions
$\checkmark$ Co-expression
$\checkmark$ Predicted
$\checkmark$ Co-localization
$\square$ Pathway
$\square$ Genetic Interactions
$\square$ Shared protein domains

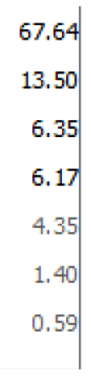

Figure 2. GeneMANIA annotation of proteins' interactions. The interaction types here are colored differently.

nodes and edges, respectively. PRDX2 is the only DEP that is present in this complex, which is PRDX2. The seed of this complex is HSPA8. The last complex includes 3 nodes, and GSTP1 is the only DEPs that is presented in this protein complex. The seed protein here is CAT.

Biological process analysis of the hub-bottlenecks and the first cluster of the main network as well could show the possible influenced groups of biological terms in the treatment of RA as shown in A and B parts of Figure 4, respectively. As it is shown in Figure 4, the Glycolytic process through fructose-6- phosphate and ATP generation from $\mathrm{ADP}$ is the most highlighted groups in hub-bottlenecks and, similarly, NADH regeneration and glyceraldehyde-3- phosphate metabolic processes are the most important ones for cluster 1 .

Since the glycolytic process through fructose-6- phosphate was the leading group in Figure 4, which was related to hub-bottlenecks, its relationships with the 3 query hubbottlenecks (GAPGH, PKM, and ENO1) are shown in Figure 5. This group via 8 terms is connected to DE hub-bottlenecks.

As it is shown in Figure 1, there are several oncogenic proteins among the added neighbors. To find the possible regulatory relationships between the oncogenic proteins and the metabolic central nodes, the action map between these elements was analyzed and shown in Figure 6. In Figure 6, AKT1, STAT3, and MYC (the 3 oncogenes) ac- 
Table 1. A list of hub-bottlenecks of the PPI network is presented. BC designates betweenness centrality and $\mathrm{K}$ represents degree value $\mathrm{a}^{\mathrm{a}, \mathrm{b}}$

\begin{tabular}{lccc}
\hline Row & Display Name & K & BC \\
\hline $\mathbf{1}$ & GAPDH $^{*}$ & 57 & 0.04 \\
$\mathbf{2}$ & ENO1* $^{*}$ & 53 & 0.03 \\
$\mathbf{3}$ & PKM $^{*}$ & 52 & 0.03 \\
$\mathbf{4}$ & PGK1 & 52 & 0.03 \\
$\mathbf{5}$ & TPI & 50 & 0.02 \\
$\mathbf{6}$ & LDHA & 48 & 0.02 \\
\hline $\mathbf{7}$ & GAPDHS & 45 & 0.02 \\
$\mathbf{8}$ & ENO2 & 42 & 0.01 \\
\hline
\end{tabular}

${ }^{\mathrm{a}}$ The proteins are ordered based on degree values.

${ }^{\mathrm{b}}$ The asterisked marked nodes are the query proteins.

\begin{tabular}{|c|c|c|c|c|}
\hline Row & Cluster & Nodes & Links & Scores \\
\hline 1 & & 31 & 457 & 30 \\
\hline 2 & & 19 & 139 & 15 \\
\hline 3 & & & 3 & \\
\hline
\end{tabular}

Figure 3. MCODE analysis of the network of thyroid cancer cells, which are treated with RA. Node score cut-offs for the complex 1, 2, and 3 are 30.467, 15.444, and 3.000, respectively. The square-shaped node indicates seed protein. Node score cut-off: 0.2 , K-core: 2, Degree cut-off: 2.

tivate GAPDH, ENO1, and PKM as the metabolic proteins. Therefore, it can be concluded that the regulation of the metabolic proteins has resulted from the regulation of oncogene proteins by RA.

\section{Discussion}

Network analysis is a useful method to screen large numbers of differential proteins for introducing a few cen- tral ones that can be used for clinical purposes. Interactome study is also a suitable method to determine new potent molecular agents that are involved in the onset, progress, and treatment of diseases. In this regard, the PPI network study is applied to discover potent proteins that are related to suppressing thyroid cancer cells by using RA. As it is shown in the result part, $8 \mathrm{DE}$ proteins, which are highlighted by a proteomic study, were candidates for exploring the most crucial ones in terms of interacting in thyroid cancer treatment by RA.

At first glance, all of the query proteins are connected directly or indirectly. GAPDH and ENO1 are connected to all of the neighbors and PKM, and ANAXA2 are linked to 5 proteins among 8 queries DE proteins. It seems that the role of these 4 proteins is prominent relative to the other ones. Centrality analysis revealed that GAPDH, ENO1, and PKM proteins are the ones with significant centrality properties known as DE hub-bottlenecks. In other words, these $3 \mathrm{DE}$ proteins not only are central proteins but also identified as top hub-bottlenecks in our network analysis. GeneMANIA analysis (Figure 2) showed the different types of relationships between the queried hub-bottlenecks. The analysis of this algorithm expresses that all the proteins tend to have similar expression as it is also indicated as downregulation in the main proteomic study. The first complex is the most worthy one in terms of a high score of interconnections and containing half of the DEPs.

The 3 query hub-bottlenecks are included in this protein complex. This finding leads to 2 valuable advantages; first, confirming the prominent role of GAPDH, ENO1, and PKM as central proteins and; second, introducing PDHB (seed of complex) as a crucial new element. PDHB gene encodes pyruvate dehydrogenase $\mathrm{E} 1$ beta subunit protein (19). Therefore, it can be inferred, as indicated in Figure 3 and Table 1, that the first cluster worth pursuing more analysis in terms of gene ontology. Figure 4 demonstrates that the high ranked cluster is significantly involved in metabolic processes. This finding also aligns with the results of the study in 2010, in which the participation of metabolic relations in thyroid cancer treated with RA was highlighted (5). This finding concludes that, as the pathway linked to the hub-bottlenecks and the most critical cluster is the same for DEPs in the first research, our outcome is valid coordination.

Moreover, the biological process analysis for the 3 down-regulated hub-bottlenecks shows that the Glycolytic process through fructose-6- phosphate is a fundamental process for these proteins. This process is also highlighted for the whole hub-bottlenecks of the main network. This indicates that the most influenced part of the metabolic system could be the Glycolytic process through fructose-6phosphate. Biological studies suggest that metabolic alter- 
A

\% Terms Per Group

Glycolytic Process Through Fructose-6Phosphate 58.82\% **

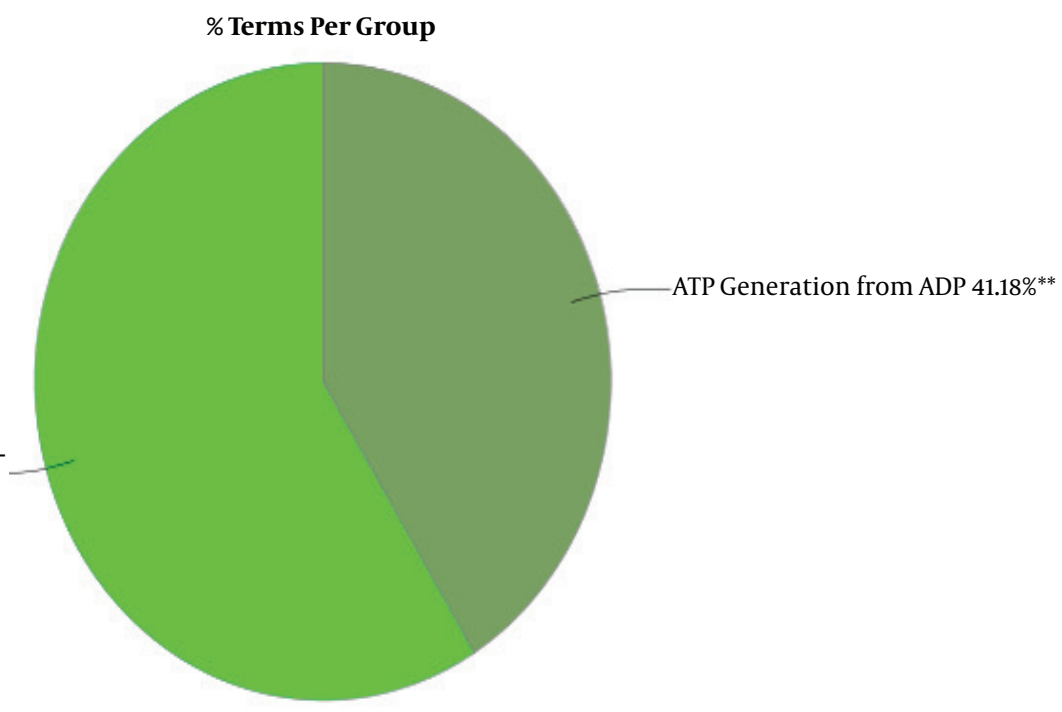

B

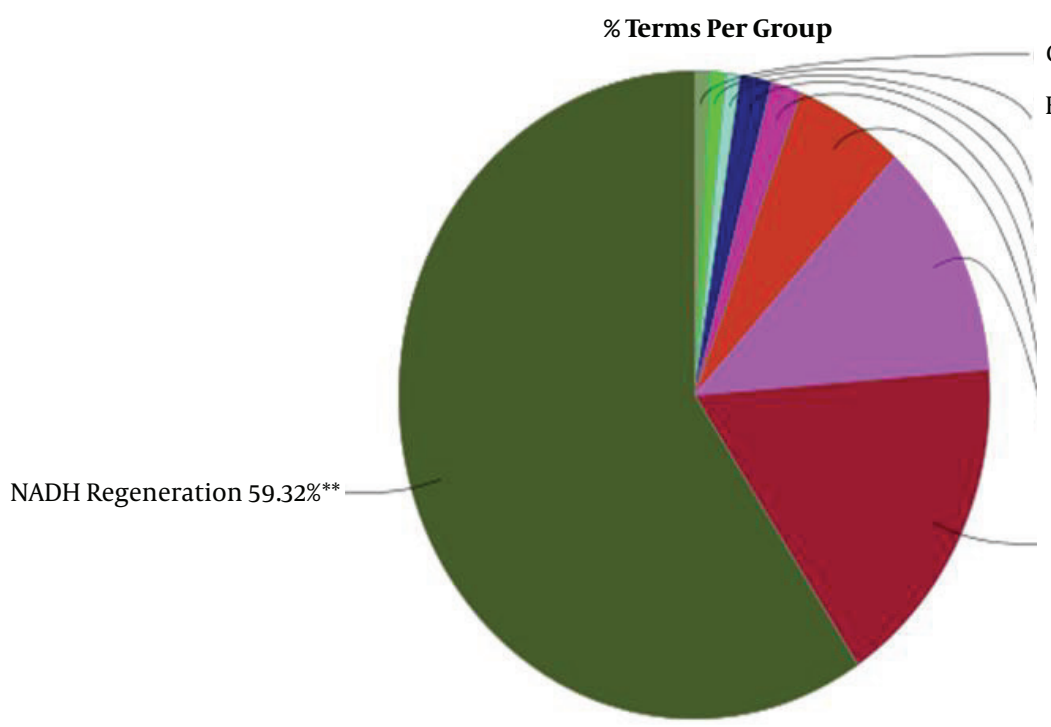

Carbohydrate Metabolic Process 0.85\%**

Fructose 6-phosphate Metabolic Process $0.85 \% * *$

Aldehyde Biosynthetic Process $0.85 \% * *$

Pentose Biosynthetic Process 1.69\% **

Fructose 1,6-bisphosphate Metabolic Process $1.69 \% * *$

Pyridine Nucleotide Metabolic Process $5.93 \%$ **

Glucose Catabolic Process 11.86\%**

Glyceraldehyde-3-phosphate Metabolic Process $16.95 \% * *$

Figure 4. Pie charts view of biological processes (BCs) evaluations of the hub-bottlenecks and the first protein complex are shown in parts A and B, respectively. The glycolytic process through fructose-6 phosphate and NADH regeneration are the primary BCs in the A and B charts, respectively.

ations, including the activation of glycolysis, are prerequisites of the promotion of aggressive cancers (20). As it is reported, cancer cells suppress the oxidation phosphorylation process in mitochondria and activate aerobic glycolysis to provide the energy required for cancer cell sur- vival and growth. It is observed that thyroid cancer cells can switch oxidation phosphorylation-glycolysis to access suitable energy levels for growing (21). Extensive alteration in the regulation of mitochondrial biochemical pathways is reported in thyroid cancer disease. The up-regulation of 


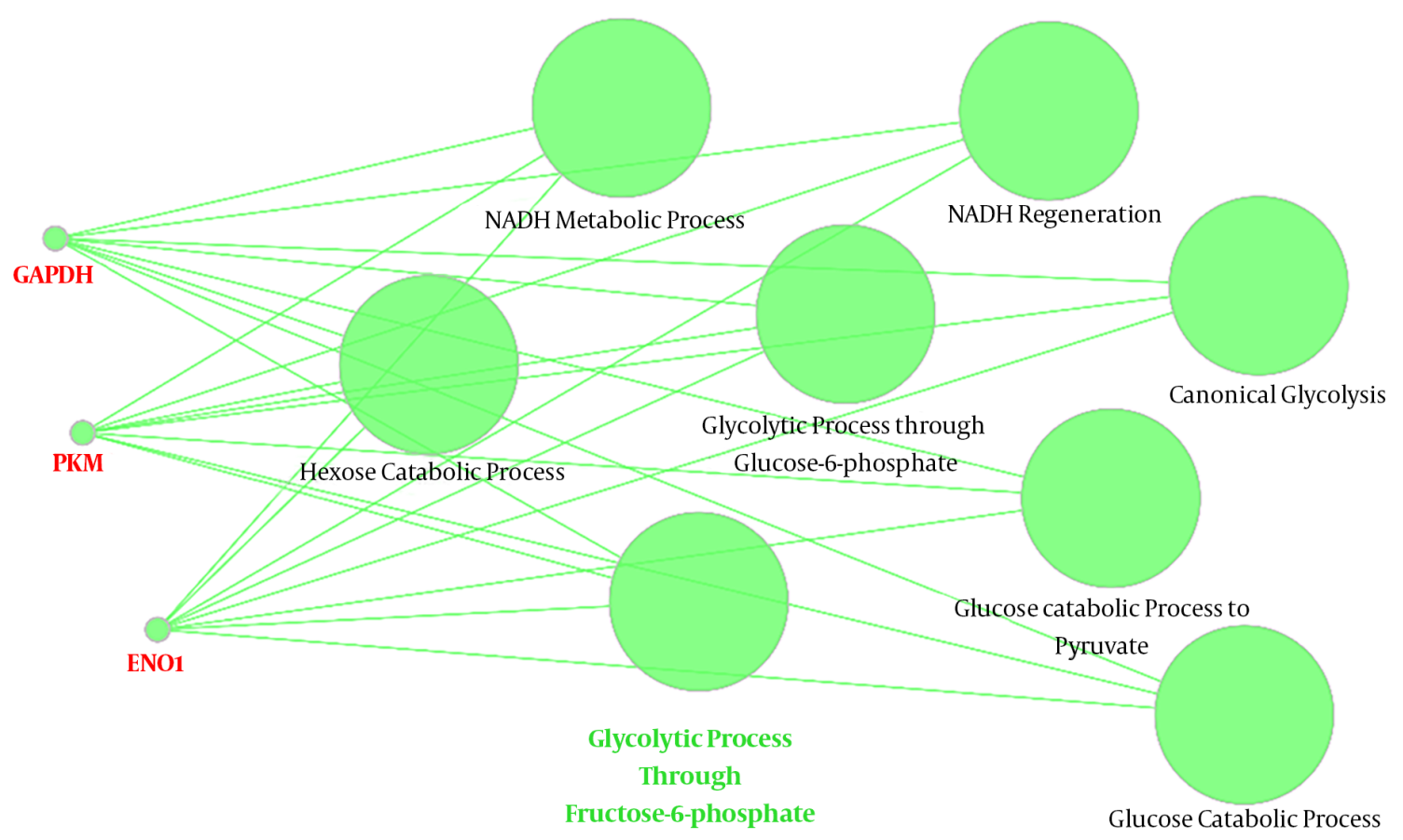

Figure 5. The network of biological terms, which are related to differentially expressed hub-bottlenecks. The terms are grouped as the glycolytic process through fructose-6phosphate.

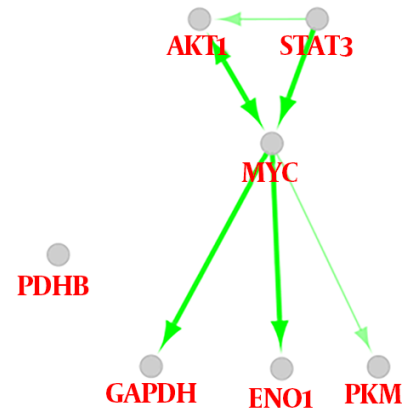

Figure 6. Activation map between metabolic central query proteins and oncogenic proteins among added neighbor proteins. PDHB as a seed protein in the main complex protein is also considered; however, it is not included in the map.

different tyrosine receptor kinase genes that elevates the relevant activities are stated (22). GAPDH as potent central DE protein in our study is a housekeeping protein (23) and such as the other identified hub-bottlenecks is not an oncogenic protein. As it is shown in Figure 1, several oncogenes are connected to the introduced central nodes. MYC and STAT3 are two oncogenes that play a critical role in differ- ent cancers. MYC plays a key role in the regulation of cancer cells' metabolism. STAT3 is a well-known oncogene that is activated in the many cancer types and is involved in the block of apoptosis $(24,25)$. The role of the other oncogene, AKT1, in the regulation of several cellular functions in various cancers is reported and discussed in detail (26).

It can be concluded that the introduced central proteins could not be the main causes of thyroid cancer and may have indirect associations. Therefore, their regulation by RA does not result in thyroid cancer suppression. It is a logical conclusion that RA regulates the adequate protein expression level of oncogenes, which involves the promotion of cancer.

The regulation of these oncogenes leads to the control of the metabolic enzymes. The action map confirms this hypothesis (Figure 6). This finding does not decrease the importance of our findings because the introduced central proteins can be useful in the follow-up of the patients.

\subsection{Conclusions}

GAPDH, ENO1, PKM, and PDHB are introduced as a consequence effect of RA on thyroid cancer cells based on network analysis. Besides, they are revealed to be related to the oncogenes such as MYC, STAT3, and AKT1. The introduced 
crucial DE proteins are linked to the term, namely the glycolytic process through fructose-6-phosphate, which is an important energy consumption style in mitochondria. Therefore, the identified critical central proteins can be used in the follow-up of the patients and monitoring of the treatment of RA used as a chemotherapy reagent.

\section{Acknowledgments}

This research is supported by the Proteomics Research Center, Shahid Beheshti University of Medical Sciences, Tehran, Iran.

\section{Footnotes}

Authors' Contribution: All the authors contributed equally to work.

Conflict of Interests: There is no conflict of interest.

Ethical Approval: The Ethical Committee of Shahid Beheshti University of Medical Sciences approved this study. The ethical code for this study is IR.SBMU.REC.1397.112.

Funding/Support: This research is supported by Proteomics Research Center, Shahid Beheshti University of Medical Sciences, Tehran, Iran.

\section{References}

1. Martinez-Aguilar J, Clifton-Bligh R, Molloy MP. Proteomics of thyroid tumours provides new insights into their molecular composition and changes associated with malignancy. Sci Rep. 2016;6:23660. doi: 10.1038/srep23660. [PubMed: 27025787]. [PubMed Central PMC4812243].

2. Wojakowska A, Chekan M, Widlak P, Pietrowska M. Application of metabolomics in thyroid cancer research. Int $J$ Endocrinol. 2015;2015:258763. doi: 10.1155/2015/258763. [PubMed: 25972898] [PubMed Central: PMC4417976].

3. Naoum GE, Morkos M, Kim B, Arafat W. Novel targeted therapies and immunotherapy for advanced thyroid cancers. Mol Cancer. 2018;17(1):51. doi: 10.1186/s12943-018-0786-0. [PubMed: 29455653] [PubMed Central: PMC5817719].

4. Liu T, Zhang W, Xiao S, Xu L, Wen Q, Bai L, et al. Mindfulness-based stress reduction in patients with differentiated thyroid cancer receiving radioactive iodine therapy: A randomized controlled trial. Cancer Manag Res. 2019;11:467-74. doi: 10.2147/CMAR.S183299. [PubMed 30655698]. [PubMed Central: PMC6324610].

5. Trojanowicz B, Sekulla C, Lorenz K, Kohrle J, Finke R, Dralle H, et al Proteomic approach reveals novel targets for retinoic acid-mediated therapy of thyroid carcinoma. Mol Cell Endocrinol. 2010;325(1-2):110-7. doi: 10.1016/j.mce.2010.05.022. [PubMed: 20538039].

6. di Masi A, Leboffe L, De Marinis E, Pagano F, Cicconi L, Rochette-Egly C, et al. Retinoic acid receptors: From molecular mechanisms to cancer therapy. Mol Aspects Med. 2015;41:1-115. doi:10.1016/j.mam.2014.12.003. [PubMed: 25543955].

7. Bashizadeh Fakhar H, Rezaie-Tavirani M, Zali H, Faraji Darkhaneh R, Kazem Nejad E, Aghazadeh MH. Proteomic analysis of low-grade serous ovarian cancer and comparing it with non-cancerous ovarian. Indi J Gynecol Oncol. 2018;16(2). doi: 10.1007/s40944-018-0187-8.
8. Barneh F, Salimi M, Goshadrou F, Ashtiani M, Mirzaie M, Zali $\mathrm{H}$, et al. Valproic acid inhibits the protective effects of stromal cells against chemotherapy in breast cancer: Insights from proteomics and systems biology. J Cell Biochem. 2018;119(11):9270-83. doi: 10.1002/jcb.27196. [PubMed: 29953653].

9. Doll S, Gnad F, Mann M. The case for proteomics and phosphoproteomics in personalized cancer medicine. Proteomics Clin Appl. 2019;13(2). e1800113. doi: 10.1002/prca.201800113. [PubMed: 30790462]. [PubMed Central: PMC6519247].

10. Safari-Alighiarloo N, Taghizadeh M, Rezaei-Tavirani M, Goliaei B, Peyvandi AA. Protein-protein interaction networks (PPI) and complex diseases. Gastroenterol Hepatol Bed Bench. 2014;7(1):17-31. [PubMed: 25436094]. [PubMed Central: PMC4017556].

11. Zali H, Zamanian-Azodi M, Rezaei Tavirani M, Akbar-Zadeh Baghban A. Protein drug targets of Lavandula angustifolia on treatment of rat alzheimer's disease. Iran J Pharm Res. 2015;14(1):291-302. [PubMed: 25561935]. [PubMed Central: PMC4277642].

12. Zamanian-Azodi M, Rezaei-Tavirani M. Investigation of health benefits of cocoa in human colorectal cancer cell line, HT-29 through interactome analysis. Gastroenterol Hepatol Bed Bench. 2019;12(1):67-73. [PubMed: 30949322]. [PubMed Central: PMC6441487].

13. Shannon P, Markiel A, Ozier O, Baliga NS, Wang JT, Ramage D, et al. Cytoscape: A software environment for integrated models of biomolecular interaction networks. Genome Res. 2003;13(11):2498504. doi: 10.1101/gr.1239303. [PubMed: 14597658]. [PubMed Central: PMC403769].

14. Szklarczyk D, Morris JH, Cook H, Kuhn M, Wyder S, Simonovic M, et al. The STRING database in 2017: Quality-controlled proteinprotein association networks, made broadly accessible. Nucleic Acids Res. 2017;45(D1):362-8. doi: 10.1093/nar/gkw937. [PubMed: 27924014]. [PubMed Central: PMC5210637].

15. Assenov Y, Ramirez F, Schelhorn SE, Lengauer T, Albrecht M. Computing topological parameters of biological networks. Bioinformatics. 2008;24(2):282-4. doi: 10.1093/bioinformatics/btm554. [PubMed: 18006545].

16. Montojo J, Zuberi K, Rodriguez H, Kazi F, Wright G, Donaldson SL, et al. GeneMANIA Cytoscape plugin: Fast gene function predictions on the desktop. Bioinformatics. 2010;26(22):2927-8. doi: 10.1093/bioinformatics/btq562. [PubMed: 20926419]. [PubMed Central: PMC2971582].

17. Bindea G, Mlecnik B, Hackl H, Charoentong P, Tosolini M, Kirilovsky A, et al. ClueGO: A cytoscape plug-in to decipher functionally grouped gene ontology and pathway annotation networks. Bioinformatics. 2009;25(8):1091-3. doi: 10.1093/bioinformatics/btp101. [PubMed: 19237447]. [PubMed Central: PMC2666812].

18. Bindea G, Galon J, Mlecnik B. CluePedia cytoscape plugin: Pathway insights using integrated experimental and in silico data. Bioinformatics. 2013;29(5):661-3. doi: 10.1093/bioinformatics/btt019. [PubMed: 23325622]. [PubMed Central: PMC3582273].

19. Brown RM, Head RA, Boubriak II, Leonard JV, Thomas NH, Brown GK. Mutations in the gene for the E1beta subunit: A novel cause of pyruvate dehydrogenase deficiency. Hum Genet. 2004;115(2):123-7. doi: 10.1007/s00439-004-1124-8. [PubMed: 15138885].

20. Lee J, Chang JY, Kang YE, Yi S, Lee MH, Joung KH, et al. Mitochondrial energy metabolism and thyroid cancers. Endocrinol Metab (Seoul). 2015;30(2):117-23. doi: 10.3803/EnM.2015.30.2.117. [PubMed: 26194071]. [PubMed Central: PMC4508255].

21. Mirebeau-Prunier D, Le Pennec S, Jacques C, Fontaine JF, Gueguen $\mathrm{N}$, Boutet-Bouzamondo $\mathrm{N}$, et al. Estrogen-related receptor alpha modulates lactate dehydrogenase activity in thyroid tumors. PLoS One. 2013;8(3). e58683. doi: 10.1371/journal.pone.0058683. [PubMed: 23516535]. [PubMed Central: PMC3596295].

22. Garcia-Rostan G, Costa AM, Pereira-Castro I, Salvatore G, Hernandez $\mathrm{R}$, Hermsem MJ, et al. Mutation of the PIK3CA gene in anaplastic thyroid cancer. Cancer Res. 2005;65(22):10199-207. doi: 10.1158/00085472.CAN-04-4259. [PubMed: 16288007]. 
23. Barber RD, Harmer DW, Coleman RA, Clark BJ. GAPDH as a housekeeping gene: analysis of GAPDH mRNA expression in a panel of $72 \mathrm{hu}-$ man tissues. Physiol Genomics. 2005;21(3):389-95. doi: 10.1152/physiolgenomics.00025.2005. [PubMed: 15769908].

24. Miller DM, Thomas SD, Islam A, Muench D, Sedoris K. c-Myc and cancer metabolism. Clin Cancer Res. 2012;18(20):5546-53. doi: 10.1158/1078-0432.CCR-12-0977. [PubMed: 23071356]. [PubMed Central: PMC3505847].
25. Bromberg JF, Wrzeszczynska MH, Devgan G, Zhao Y, Pestell RG, Albanese C, et al. Stat3 as an oncogene. Cell. 1999;98(3):295-303. doi 10.1016/s0092-8674(00)81959-5. [PubMed:10458605].

26. Juntilla MM, Patil VD, Calamito M, Joshi RP, Birnbaum MJ, Koretzky GA. AKT1 and AKT2 maintain hematopoietic stem cell function by regulating reactive oxygen species. Blood. 2010;115(20):4030-8. doi: 10.1182/blood-2009-09-241000. [PubMed: 20354168]. [PubMed Central: PMC2875090]. 